\title{
Mesh patterns and the expansion of permutation statistics as sums of permutation patterns
}

\author{
Petter Brändén* \\ Department of Mathematics, Stockholm University, \\ SE-106 91 Stockholm, Sweden \\ Anders Claesson ${ }^{\dagger}$ \\ Department of Computer and Information Sciences, \\ University of Strathclyde, Glasgow, G1 1XH, UK \\ Submitted: Feb 21, 2011; Accepted: Mar 3, 2011; Published: Mar 15, 2011 \\ Mathematics Subject Classification: 05A05, 05A15, 05A19
}

Dedicated to Doron Zeilberger on the occasion of his sixtieth birthday

\begin{abstract}
Any permutation statistic $f: \mathfrak{S} \rightarrow \mathbb{C}$ may be represented uniquely as a, possibly infinite, linear combination of (classical) permutation patterns: $f=\Sigma_{\tau} \lambda_{f}(\tau) \tau$. To provide explicit expansions for certain statistics, we introduce a new type of permutation patterns that we call mesh patterns. Intuitively, an occurrence of the mesh pattern $p=(\pi, R)$ is an occurrence of the permutation pattern $\pi$ with additional restrictions specified by $R$ on the relative position of the entries of the occurrence. We show that, for any mesh pattern $p=(\pi, R)$, we have $\lambda_{p}(\tau)=(-1)^{|\tau|-|\pi|} p^{\star}(\tau)$ where $p^{\star}=\left(\pi, R^{c}\right)$ is the mesh pattern with the same underlying permutation as $p$ but with complementary restrictions. We use this result to expand some well known permutation statistics, such as the number of left-to-right maxima, descents, excedances, fixed points, strong fixed points, and the major index. We also show that alternating permutations, André permutations of the first kind and simsun permutations occur naturally as permutations avoiding certain mesh patterns. Finally, we provide new natural Mahonian statistics.
\end{abstract}

${ }^{*} \mathrm{~PB}$ is a Royal Swedish Academy of Sciences Research Fellow supported by a grant from the Knut and Alice Wallenberg Foundation.

${ }^{\dagger}$ AC was supported by grant no. 090038011 from the Icelandic Research Fund. 


\section{Introduction}

\subsection{Mesh patterns}

Let $[a, b]$ be the integer interval $\{i \in \mathbb{Z}: a \leq i \leq b\}$. Denote by $\mathfrak{S}_{n}$ the set of permutations of $[1, n]$. A mesh pattern is a pair

$$
p=(\pi, R) \text { with } \pi \in \mathfrak{S}_{k} \text { and } R \subseteq[0, k] \times[0, k] .
$$

An example is $p=(3241,\{(0,2),(1,3),(1,4),(4,2),(4,3)\})$. To depict this mesh pattern we plot the points $(i, \pi(i))$ in a Cartesian coordinate system, and for each $(i, j) \in R$ we shade the unit square with bottom left corner $(i, j)$ :

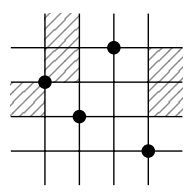

Let $p=(\pi, R)$ be a mesh pattern with $k=|\pi|$, where $|\pi|$ denotes the number of letters in $\pi$, and let $\tau \in \mathfrak{S}_{n}$. We will think of $p$ as a function on permutations that counts occurrences of $p$. Intuitively, $p(\tau)$ is the number of "classical" occurrences of $\pi$ in $\tau$ with additional restrictions on the relative position of the entries of the occurrence of $\pi$ in $\tau$. These restrictions say that no elements of $\tau$ are allowed in the shaded regions of the figure above. Formally, an occurrence of $p$ in $\tau$ is a subset $\omega$ of the plot of $\tau, G(\tau)=\{(i, \tau(i))$ : $i \in[1, n]\}$, such that there are order-preserving injections $\alpha, \beta:[1, k] \rightarrow[1, n]$ satisfying two conditions that we shall now describe. The first condition is that $\omega$ is an occurrence of $\pi$ in the classical sense. That is,

(i) $\omega=\{(\alpha(i), \beta(j)):(i, j) \in G(\pi)\}$.

Define $R_{i j}=[\alpha(i)+1, \alpha(i+1)-1] \times[\beta(j)+1, \beta(j+1)-1]$ for $i, j \in[0, k]$, where $\alpha(0)=\beta(0)=0$ and $\alpha(k+1)=\beta(k+1)=n+1$. Then the second condition is

(ii) if $(i, j) \in R$ then $R_{i j} \cap G(\tau)=\emptyset$.

Classical [10], vincular [2] and bivincular [4] patterns can all be seen as special mesh patterns: $p=(\pi, R)$ is a classical pattern if $R=\emptyset ; p$ is a vincular pattern if $R$ is a union of vertical strips, $\{i\} \times[0,|\pi|] ; p$ is a bivincular pattern if $R$ is a union of vertical strips and horizontal strips, $[0,|\pi|] \times\{i\}$. An example is provided by the following bivincular pattern which has been studied by Bousquet-Mélou et al. [4]:

$$
(231,[0,3] \times\{1\} \cup\{1\} \times[0,3])=\text {. }
$$

It is also easy to write any barred pattern [14] with only one barred letter as a mesh pattern. Indeed, if $\pi(i)$ is the only barred letter of a given barred pattern $\pi$, then the corresponding mesh pattern is $\left(\pi^{\prime},\{(i-1, \pi(i)-1)\}\right.$, where $\pi^{\prime}$ is obtained from $\pi$ by 
removing $\pi(i)$ and subtracting one from each letter that is larger than $\pi(i)$. For instance, West [14] characterized the permutations sortable by two passes through a stack as those that avoid the classical pattern 2341 and the barred pattern $3 \overline{5} 241$. So, in terms of mesh patterns, it is the set of permutations that avoid

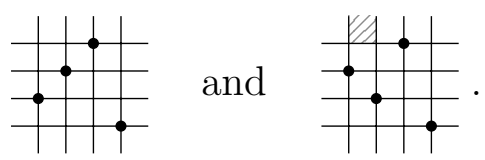

The number of saturated chains in Young's Lattice from $0 \hat{0}$ (the empty partition) to a partition $\lambda$ is the number of standard Young tableaux of shape $\lambda$, and the total number of saturated chains from $\hat{0}$ to rank $n$ is the number of involutions in $\mathfrak{S}_{n}$. Bergeron et al. [3] studied a composition analogue of Young's lattice. They gave an embedding of the saturated chains from $\hat{0}$ to rank $n$ into $\mathfrak{S}_{n}$, and they characterized the image under this embedding as follows: Let $T(\pi)$ be the increasing binary tree corresponding to $\pi{ }^{1}$ Then $\pi \in \mathfrak{S}_{n}$ encodes a saturated chain from $\hat{0}$ to rank $n$ if and only if for any vertex $v$ of $T(\pi)$ that do not belong to the leftmost branch of $T(\pi)$ and has two sons, the label of the left son is less that the label of the right son. There is a unique smallest permutation not satisfying this, namely 1423; the corresponding increasing binary tree is

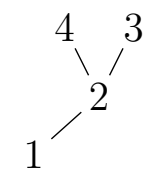

In terms of mesh patterns the permutations encoding saturated chains from $\hat{0}$ to rank $n$ are precisely those that avoid

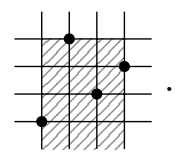

By $p(\tau)$ we shall denote the number of occurrences of $p$ in $\tau$, thus regarding $p$ as a function from $\mathfrak{S}=\cup_{n \geq 0} \mathfrak{S}_{n}$ to $\mathbb{N}$. We will now explain how a few well known permutation statistics may be expressed in terms of mesh patterns. A left-to-right maximum of $\tau$ is an index $j$ such that $\tau(i)<\tau(j)$ for $i<j$. We write $\operatorname{lmax}(\tau)$ for the number of left-to-right maxima in $\tau$. A descent is an $i$ such that $\tau(i)>\tau(i+1)$. The number of descents is denoted $\operatorname{des}(\tau)$. An inversion is a pair $i<j$ such that $\tau(i)>\tau(j)$. The number of inversions is denoted $\operatorname{INv}(\tau)$. For permutations $\alpha$ and $\beta$, let their direct sum be $\alpha \oplus \beta=\alpha \beta^{\prime}$, where $\beta^{\prime}$ is obtained from $\beta$ by adding $|\alpha|$ to each of its letters, and juxtaposition denotes concatenation. We say that $\tau$ has $k$ components, and write $\operatorname{comp}(\tau)=k$, if $\tau$ is the direct sum of $k$, but not $k+1$, non-empty permutations. We have

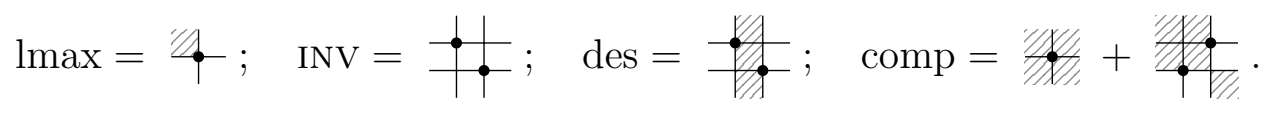

\footnotetext{
${ }^{1}$ If $\pi$ is the empty word then $T(\pi)$ is the empty tree. Otherwise, write $\pi=\sigma a \tau$ with $a=\min (\pi)$, then $T(\pi)$ is the binary tree with root $a$ attached to a left subtree $T(\sigma)$ and a right subtree $T(\tau)$.
} 


\subsection{Permutation statistics and an incidence algebra}

In what follows we will often simply write $\pi$ instead of $(\pi, \emptyset)$, so INV $=21$. We shall see that any function stat : $\mathfrak{S} \rightarrow \mathbb{C}$ may be represented uniquely as a (possibly infinite) sum stat $=\sum_{\pi \in \mathfrak{S}} \lambda(\pi) \pi$, where $\{\lambda(\pi)\}_{\pi \in \mathfrak{S}} \subset \mathbb{C}$.

Let $Q$ be a locally finite poset, and let $\operatorname{Int}(Q)=\{(x, y) \in Q \times Q: x \leq y\}$. Recall that the incidence algebra, $I(Q)$, of $(Q, \leq)$ over $\mathbb{C}$ is the $\mathbb{C}$-algebra of all functions $F$ : $\operatorname{Int}(Q) \rightarrow \mathbb{C}$ with multiplication (convolution) defined by

$$
(F G)(x, z)=\sum_{x \leq y \leq z} F(x, y) G(y, z),
$$

and identity, $\delta$, defined by $\delta(x, y)=1$ if $x=y$, and $\delta(x, y)=0$ if $x \neq y$; see for example [11, Sec. 3.6].

Define a partial order on $\mathfrak{S}$ by $\pi \leq \sigma$ in $\mathfrak{S}$ if $\pi(\sigma)>0$. Define $P \in I(\mathfrak{S})$ by

$$
P(\pi, \sigma)=\pi(\sigma) .
$$

Note that $P$ is invertible because $P(\pi, \pi)=1$, see [11, Prop. 3.6.2]. Therefore, for any permutation statistic, stat $: \mathfrak{S} \rightarrow \mathbb{C}$, there are unique scalars $\{\lambda(\sigma)\}_{\sigma \in \mathfrak{S}} \subset \mathbb{C}$ such that

$$
\text { stat }=\sum_{\sigma \in \mathfrak{S}} \lambda(\sigma) \sigma .
$$

In other words, any permutation statistic can be written as a unique, typically infinite, formal linear combination of (classical) patterns. Indeed, $I(\mathfrak{S})$ acts on the right of $\mathbb{C}^{\mathfrak{S}}$ by

$$
(f * F)(\pi)=\sum_{\sigma \leq \pi} f(\sigma) F(\sigma, \pi) .
$$

Thus (1) is equivalent to stat $=\lambda * P$ and, since $P$ is invertible, $\lambda=$ stat $* P^{-1}$.

\section{The Reciprocity Theorem}

The following mysterious looking identity for the descent statistic

$$
\operatorname{des}=\sum_{\substack{\pi \in \mathfrak{S} \\ \pi(1)>\pi(|\pi|)}}(-1)^{|\pi|} \pi
$$

is an instance of what we call the Reciprocity Theorem for mesh patterns. It tells us what the coefficients $\{\lambda(\sigma)\}_{\sigma \in \mathfrak{S}}$ are in the special case when stat $=p$, a mesh pattern. The Reciprocity Theorem may be viewed as a justification for the introduction of mesh patterns. Indeed it shows that to describe the coefficients of "generalized permutation patterns" requires that the set of patterns is closed under taking complementary restrictions. 
Theorem 1 (Reciprocity). Let $p=(\pi, R)$ be a mesh pattern and let $p^{\star}=\left(\pi, R^{c}\right)$, where $R^{c}=[0,|\pi|]^{2} \backslash R$. Then

$$
p=\sum_{\sigma \in \mathfrak{S}} \lambda(\sigma) \sigma, \text { where } \lambda(\sigma)=(-1)^{|\sigma|-|\pi|} p^{\star}(\sigma) .
$$

Proof. We need to prove that $p^{\star}(\tau)=\sum_{\sigma \leq \tau}(-1)^{|\pi|-|\sigma|} p(\sigma) \sigma(\tau)$ for all $\tau \in \mathfrak{S}$. We will think of an occurrence of a pattern $p$ in $\sigma$ as the corresponding subword of $\sigma$. The right-hand side may be written as

$$
\sum_{\left(\omega_{\pi}, \omega_{\sigma}\right)}(-1)^{|\pi|-|\sigma|}
$$

in which sum is over all pairs $\left(\omega_{\pi}, \omega_{\sigma}\right)$ where $\omega_{\pi}$ is a occurrence of $p$ in $\omega_{\sigma}$ and $\omega_{\sigma}$ is an occurrence of some $\sigma \leq \tau$. Expression (2) may, in turn, be written as

$$
\sum_{\omega_{\pi}}(-1)^{|\pi|} \mu\left(\omega_{\pi}\right)
$$

where $\mu\left(\omega_{\pi}\right)$ is the contribution from a given occurrence $\omega_{\pi}$ of $\pi$. Given $\omega_{\pi}$, to create a pair $\left(\omega_{\pi}, \omega_{\sigma}\right)$ we include any elements which are in squares not indexed by the restrictions $R$. Let $X\left(\omega_{\pi}\right)$ be the set of such elements. Hence

$$
\mu\left(\omega_{\pi}\right)=\sum_{S \subseteq X\left(\omega_{\pi}\right)}(-1)^{|\pi|+|S|} .
$$

Thus $\mu\left(\omega_{\pi}\right)=0$ unless $X\left(\omega_{\pi}\right)=\emptyset$. Clearly $X\left(\omega_{\pi}\right)=\emptyset$ if and only if $\omega_{\pi}=\omega_{\sigma}$ and $\omega_{\sigma}$ is an occurrence of $p^{\star}$. Consequently, $\sum_{\omega_{\pi}}(-1)^{|\pi|} \mu\left(\omega_{\pi}\right)=p^{\star}(\tau)$, as claimed.

Corollary 2 (Inverse Theorem). The inverse of $P$ in $I(\mathfrak{S})$ is given by

$$
P^{-1}(\pi, \tau)=(-1)^{|\tau|-|\pi|} P(\pi, \tau) .
$$

Equivalently, if $f, g: \mathfrak{S} \rightarrow \mathbb{C}$, then

$$
f(\pi)=\sum_{\sigma \leq \pi} g(\sigma) \sigma(\pi), \quad \text { for all } \pi \in \mathfrak{S}
$$

if and only if

$$
g(\pi)=\sum_{\sigma \leq \pi} f(\sigma)(-1)^{|\pi|-|\sigma|} \sigma(\pi), \quad \text { for all } \pi \in \mathfrak{S} .
$$

Proof. For $\pi \in \mathfrak{S}_{k}$, let $p=(\pi,[0, k] \times[0, k])$. Then $p^{\star}=(\pi, \emptyset)$ and $p(\tau)=\delta(\pi, \tau)$, so by the Reciprocity Theorem,

$$
\delta(\pi, \tau)=\sum_{\pi \leq \sigma \leq \tau}(-1)^{|\sigma|-|\pi|} P(\pi, \sigma) P(\sigma, \tau),
$$

from which the result follows. 


\section{Expansions of some permutation statistics}

Babson and Steingrímsson's [2] classification of Mahonian statistics is in terms of vincular patterns. For example, the major index, MAJ, can be defined as

$$
(21,\{1\} \times[0,2])+(132,\{2\} \times[0,3])+(231,\{2\} \times[0,3])+(321,\{2\} \times[0,3]),
$$

or in pictures:

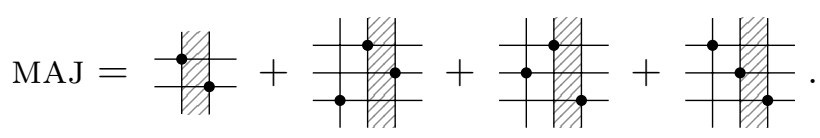

By the Reciprocity Theorem we may represent the major index as MAJ $=\sum_{\pi \in \mathfrak{S}} \lambda(\pi) \pi$ where

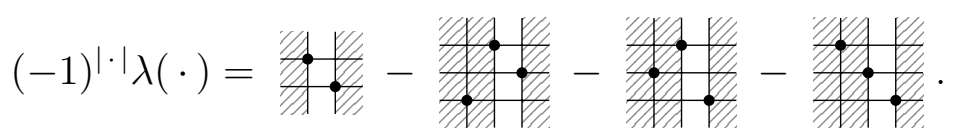

This last expression simplifies to

$$
\lambda(\pi)= \begin{cases}1 & \text { if } \pi=21, \\ (-1)^{n} & \text { if } \pi(2)<\pi(n)<\pi(1), \\ (-1)^{n+1} & \text { if } \pi(1)<\pi(n)<\pi(2), \\ 0 & \text { otherwise, }\end{cases}
$$

where $n=|\pi|$.

Now, let us plot the values of $\pi \in \mathfrak{S}$ in a Cartesian coordinate system and locate the position of $x=\pi(j)$ :

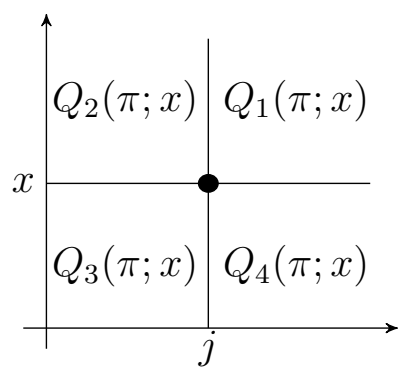

Here $Q_{2}(\pi ; x)=\{\pi(i): i<j$ and $\pi(i)>x\}$, and the sets $Q_{k}(\pi ; x)$ for $k=1,3,4$ are defined similarly. Many permutation patterns are defined in terms of the $Q_{k}$ 's.

- $x$ is a left-to-right maximum if $Q_{2}(\pi ; x)=\emptyset$. Recall that $\operatorname{lmax}(\pi)$ denotes the number of left-to-right maxima in $\pi$;

- $x$ is a fixed point if $\left|Q_{2}(\pi ; x)\right|=\left|Q_{4}(\pi ; x)\right|$. Denote by fix $(\pi)$ the number of fixed points in $\pi$; 
- The excess of $x$ in $\pi$ is $x-j=\left|Q_{4}(\pi ; x)\right|-\left|Q_{2}(\pi ; x)\right|$. For $k \in \mathbb{Z}$, let $\operatorname{exc}_{k}(\pi)$ be the number of $x$ in $\pi$ for which $\left|Q_{4}(\pi ; x)\right|-\left|Q_{2}(\pi ; x)\right|=k$;

- $x$ is an excedance top if $\left|Q_{4}(\pi ; x)\right|>\left|Q_{2}(\pi ; x)\right|$. Denote by $\operatorname{exc}(\pi)$ the number of excedance tops in $\pi$;

- $x$ is a strong fixed point if $Q_{2}(\pi ; x)=Q_{4}(\pi ; x)=\emptyset$, see [11, Ex. 1.32b]. Denote by $\operatorname{six}(\pi)$ the number of strong fixed points in $\pi$;

- $x$ is a skew strong fixed point if $Q_{1}(\pi ; x)=Q_{3}(\pi ; x)=\emptyset$. Denote by $\operatorname{ssfix}(\pi)$ the number of skew strong fixed points in $\pi$. Moreover, let $\operatorname{SSF}(\pi)$ be the set of skew strong fixed points in $\pi$.

\section{Proposition 3.}

$$
\operatorname{lmax}=\sum_{\substack{\pi \in \mathfrak{S} \\ \pi(|\pi|)=1}}(-1)^{|\pi|-1} \pi
$$

Proof. The result follows from the Reciprocity Theorem, as the function $\pi \mapsto \chi(\pi(|\pi|)=$

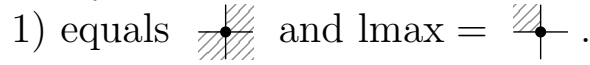

Proposition 4. Let $k \in \mathbb{Z}$. Then

$$
\operatorname{exc}_{k}=\sum_{\pi}\left((-1)^{|\pi|-k-1} \sum_{x \in \operatorname{SSF}(\pi)}\left(\begin{array}{c}
|\pi|-1 \\
x-k-1
\end{array}\right)\right) \pi .
$$

In particular

$$
\text { fix }=\sum_{\pi}\left((-1)^{|\pi|-1} \sum_{x \in \operatorname{SSF}(\pi)}\left(\begin{array}{c}
|\pi|-1 \\
x-1
\end{array}\right)\right) \pi
$$

and

$$
\operatorname{exc}=\sum_{\pi}\left((-1)^{|\pi|-2} \sum_{x \in \operatorname{SSF}(\pi)}\left(\begin{array}{c}
|\pi|-2 \\
x-2
\end{array}\right)\right) \pi .
$$

Proof. By the Inverse Theorem, $\operatorname{exc}_{k}=\sum_{\pi} \lambda_{k}(\pi) \pi$ where

$$
\lambda_{k}(\pi)=\sum_{\sigma \leq \pi}(-1)^{|\pi|-|\sigma|} \operatorname{exc}_{k}(\sigma) \sigma(\pi) .
$$

Let $\Omega_{k}(\pi)$ be the set of pair $(x, \omega)$ such that $\omega$ is a subword of $\pi$ and $x$ is a letter of $\omega$ that has excess $k$ in $\omega$. Let $\operatorname{alph}(\omega)$ denote the set of letters in $\omega$. Note that $(x, \omega) \in \Omega_{k}(\pi)$ if and only if $\left|Q_{2}(\pi ; x) \cap \operatorname{alph}(\omega)\right|+k=\left|Q_{4}(\pi ; x) \cap \operatorname{alph}(\omega)\right|$. Let $\alpha(x)=$ $\min \left(Q_{1}(\pi ; x) \cup Q_{3}(\pi ; x)\right)$, where $\min (\emptyset)=\infty$, and define an involution $\Psi: \Omega_{k}(\pi) \rightarrow$ $\Omega_{k}(\pi)$ by

$$
\Psi(x, \omega)= \begin{cases}(x, \omega) & \text { if } \alpha(x)=\infty, \\ (x, \omega \backslash \alpha(x)) & \text { if } \alpha(x) \in \operatorname{alph}(\omega), \\ (x, \omega \cup \alpha(x)) & \text { otherwise. }\end{cases}
$$


Here $\omega \backslash \alpha(x)$ denotes the word obtained by deleting $\alpha(x)$, and $\omega \cup \alpha(x)$ the subword of $\pi$ obtained by adding $\alpha(x)$ to $\omega$ at the correct position. The mapping $\Psi$ is well-defined since the property of $x$ having excess $k$ is invariant under adding elements to $Q_{1}(x)$ and $Q_{3}(x)$. Also, $\Psi$ reverses the sign, $(-1)^{|\pi|-|\omega|}$, on non fixed points. Moreover, $(x, \omega)$ is a fixed point if and only if $Q_{1}(x)=Q_{3}(x)=\emptyset$, that is, if and only if $x$ is a skew strong fixed point of $\pi$. It remains to determine the contribution of the skew strong fixed points $x$ :

$$
\begin{aligned}
\sum_{\substack{(x, \omega) \in \Omega_{k}(\pi) \\
x \in \operatorname{SSF}(\pi)}}(-1)^{|\pi|-|\omega|} & =\sum_{j}\left(\begin{array}{c}
n-x \\
j
\end{array}\right)\left(\begin{array}{c}
x-1 \\
j+k
\end{array}\right)(-1)^{|\pi|-2 j-k-1} \\
& =(-1)^{|\pi|-k-1} \sum_{j}\left(\begin{array}{c}
n-x \\
j
\end{array}\right)\left(\begin{array}{c}
x-1 \\
j+k
\end{array}\right) .
\end{aligned}
$$

Hence

$$
(-1)^{|\pi|-k-1} \lambda_{k}(\pi)=\sum_{x \in \operatorname{SSF}(\pi)} \sum_{j}\left(\begin{array}{c}
n-x \\
j
\end{array}\right)\left(\begin{array}{c}
x-1 \\
j+k
\end{array}\right)=\sum_{x \in \operatorname{SSF}(\pi)}\left(\begin{array}{c}
|\pi|-1 \\
x-k-1
\end{array}\right),
$$

as claimed. The coefficient in front of $\pi$ in the expansion of exc is $\sum_{k \geq 1} \lambda_{k}(\pi)$. The expansion of exc then follows from

$$
\sum_{j=0}^{k}(-1)^{j}\left(\begin{array}{l}
n \\
j
\end{array}\right)=(-1)^{k}\left(\begin{array}{c}
n-1 \\
k
\end{array}\right)
$$

\section{Proposition 5.}

$$
\operatorname{sfix}=\sum_{\pi}(-1)^{|\pi|-1} \operatorname{ssfix}(\pi) \pi
$$

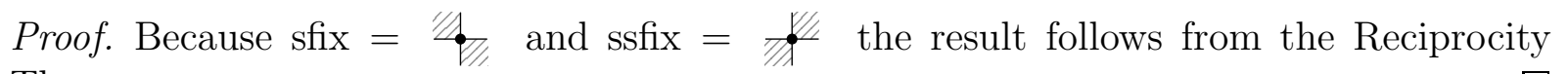
Theorem.

\section{Euler numbers}

A permutation $\pi \in \mathfrak{S}_{n}$ is said to be alternating if

$$
\pi(1)>\pi(2)<\pi(3)>\pi(4)<\cdots
$$

Clearly the set of alternating permutations are exactly the permutations that avoid the vincular/mesh patterns

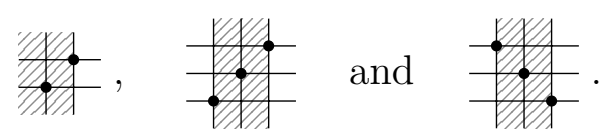


In 1879, André [1] showed that the number of alternating permutations in $\mathfrak{S}_{n}$ is the Euler number $E_{n}$ given by

$$
\sum_{n \geq 0} E_{n} x^{n} / n !=\sec x+\tan x .
$$

There are several other sets of permutations enumerated by the Euler numbers, see [12]. A simsun permutation may be defined as a permutation $\pi \in \mathfrak{S}_{n}$ for which for all $1 \leq i \leq n$, after removing the $i$ largest letters of $\pi$, the remaining word has no double descents. In terms of mesh patterns, a permutation is simsun if and only if it avoids the pattern

$$
\mathfrak{s i m s u n}=\frac{-1}{\text { t }} \text {. }
$$

simsun permutations are central in describing the action of the symmetric group on the maximal chains of the partition lattice, and the number of simsun permutations in $\mathfrak{S}_{n}$ is the Euler number $E_{n+1}$, see [13].

Another important class of permutations counted by the Euler numbers are the André permutations of various kinds introduced by Foata and Schützenberger [6] and further studied by Foata and Strehl [7]. If $\pi \in \mathfrak{S}_{n}$ and $x=\pi(i) \in[1, n]$ let $\lambda(x), \rho(x) \subset[1, n]$ be defined as follows. Let $\pi(0)=\pi(n+1)=-\infty$.

- $\lambda(x)=\left\{\pi(k): j_{0}<k<i\right\}$ where $j_{0}=\max \{j: j<i$ and $\pi(j)<\pi(i)\}$, and

- $\rho(x)=\left\{\pi(k): i<k<j_{1}\right\}$ where $j_{1}=\min \{j: i<j$ and $\pi(j)<\pi(i)\}$.

A permutation $\pi \in \mathfrak{S}_{n}$ is an André permutation of the first kind if

$$
\max \lambda(x) \leq \max \rho(x)
$$

for all $x \in[1, n]$, where $\max \emptyset=-\infty$. In particular, $\pi$ has no double descents and $\pi(n-$ $1)<\pi(n)=n$. The concept of André permutations of the first kind extends naturally to permutation of any finite totally ordered set. The following recursive description of André permutations of the first kind follows immediately from the definition.

Lemma 6. Let $\pi \in \mathfrak{S}_{n}$ be such that $\pi(n)=n$. Write $\pi$ as the concatenation $\pi=L 1 R$. Then $\pi$ is an André permutation of the first kind if and only if $L$ and $R$ are André permutations of the first kind.

Theorem 7. Let $\pi \in \mathfrak{S}_{n}$. Then $\pi$ is an André permutation of the first kind if and only if it avoids

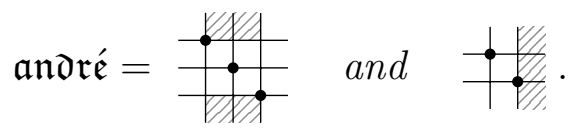

Proof. Note that $\pi \in \mathfrak{S}_{n}$ avoids the second pattern if and only if $\pi(n)=n$. Write $\pi$ as $\pi=L 1 R$. Then $\pi$ avoids andré if and only if $L$ and $R$ avoid the two patterns. Thus the set of all permutations that avoid the two patterns have the same recursive description as the set of all André permutation of the first kind, and hence the sets agree. 
Corollary 8. $\left|\mathfrak{S}_{n}(\mathfrak{a n d} \mathfrak{d} \mathfrak{e})\right|=E_{n+1}$.

Note that Lemma 6 immediately implies a version of the recursion formula for the Euler numbers

$$
E_{n+1}=\sum_{k=0}^{n-1}\left(\begin{array}{c}
n-1 \\
k
\end{array}\right) E_{k+1} E_{n-k-1}
$$

where $E_{0}=1$.

Using a computer, it is not hard to see that up to trivial symmetries the only essentially different mesh patterns $p=(321, R)$ such that $\left|\mathfrak{S}_{n}(p)\right|=E_{n+1}$ for all $n$ are $\mathfrak{s i m s u n}$ and andré.

\section{New Mahonian Statistics}

There are many ways of expressing the permutation statistic INV as a sum of mesh patterns. For instance,

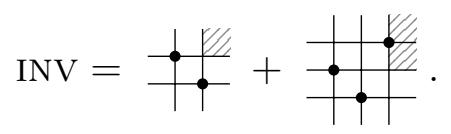

Indeed, given $\pi \in \mathfrak{S}_{n}$ we may partition the set of inversions ${ }^{2}$ of $\pi$ into two sets as follows. Let $I^{+}(\pi)$ denote the set of inversions that play the role of 21 in some occurrence of 213 , and let $I^{-}(\pi)$ denote the set of inversions that do not play the role of 21 in any occurrence of 213. Then the first pattern in the right-hand-side of (3) agrees with $\pi \mapsto\left|I^{-}(\pi)\right|$, and the second with $\pi \mapsto\left|I^{+}(\pi)\right|$.

There is a similar decomposition of non-inversions:

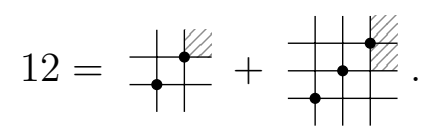

Now $A^{+}(\pi)$ is the set of non-inversions that play the role of 12 in some occurrence of 123 , and $A^{-}(\pi)$ is the set of non-inversions that do not play the role of 12 in any occurrence of 123 .

Can we mix the patterns in (3) and (4) and still get a Mahonian statistic? Let

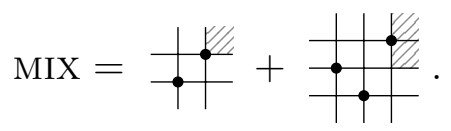

We will prove that MIX is Mahonian. Since $\operatorname{MIX}(\pi)=\left|A^{-}(\pi)\right|+\left|I^{+}(\pi)\right|$ and $12(\pi)=$ $\left|A^{-}(\pi)\right|+\left|A^{+}(\pi)\right|$ it suffices to find a bijection $\psi$ that fixes $\left|A^{-}(\pi)\right|$ and is such that $\left|A^{+}(\psi(\pi))\right|=\left|I^{+}(\pi)\right|$. In fact we will prove more. Let $M, I \subseteq[n]$ be such that $|M|=|I|$ and $n \in M \cap I$, and let $\mathfrak{S}_{n}(M, I)$ be the set of permutations in $\mathfrak{S}_{n}$ that have right-to-left maxima exactly at the positions indexed by $I$, and set of values of the right-to-left maxima

\footnotetext{
${ }^{2}$ Here the set of inversions means the set of occurrences of the pattern 21, not the positions of the inversions.
} 
equal to $M$. Let $\pi \in \mathfrak{S}_{n}(M, I)$, and define two functions $\mathrm{CI}(\pi), \mathrm{CA}(\pi):[n] \backslash M \rightarrow[n-1]$ by

$$
\begin{array}{rlrl}
\operatorname{CI}(\pi)(y) & =\left|I_{y}^{+}\right|, & & \text {where } I_{y}^{+}=\left\{x:(y, x) \in I^{+}(\pi)\right\}, \text { and } \\
\operatorname{CA}(\pi)(y) & =\left|A_{y}^{+}\right|, & \text {where } A_{y}^{+}=\left\{x:(x, y) \in A^{+}(\pi)\right\} .
\end{array}
$$

Lemma 9. Let $M, I \subseteq[n]$ be such that $|M|=|I|$ and $n \in M \cap I$. Then

$$
\mathrm{CI}(\pi)=\mathrm{CI}(\sigma) \text { if and only if } \pi=\sigma,
$$

and

$$
\mathrm{CA}(\pi)=\mathrm{CA}(\sigma) \text { if and only if } \pi=\sigma
$$

for all $\pi, \sigma \in \mathfrak{S}_{n}(M, I)$.

Proof. Suppose that we know CI $(\pi)$, and let $[n] \backslash M=\left\{s_{1}<\cdots<s_{k}\right\}$. Then $\operatorname{CI}(\pi)\left(s_{k}\right)$ tells us the position of $s_{k}$ in $\pi$, and recursively we can read off the position of $s_{i}$ from $\mathrm{CI}(\pi)\left(s_{i}\right)$, given that we know the positions of $s_{i+1}, \ldots, s_{k}$. Hence we can reconstruct $\pi$ from $\mathrm{CI}(\pi)$.

Suppose that $s_{1}, \ldots, s_{j}$ are the elements of $[n] \backslash M$ that are smaller than $\min (M)$. Then $\mathrm{CA}(\pi)\left(s_{j}\right)$ tells us the position of $s_{j}$ in $\pi$, and recursively we can read off the position of $s_{i}$ from $\mathrm{CA}(\pi)\left(s_{i}\right)$, given that we know the positions of $s_{i+1}, \ldots, s_{j}$. We can continue in the same way to read off the positions of the elements of $[n] \backslash M$ that are between $\min (M)$ and $\min (M \backslash\{\min (M)\})$ in size. Continuing this procedure we will recover $\pi$ from $\mathrm{CA}(\pi)$.

Theorem 10. Let $M, I \subseteq[n]$ be such that $|M|=|I|$ and $n \in M \cap I$. There is an involution $\psi: \mathfrak{S}_{n}(M, I) \rightarrow \mathfrak{S}_{n}(M, I)$ such that

$$
(\mathrm{CA}(\pi), \mathrm{CI}(\pi))=(\mathrm{CI}(\psi(\pi)), \mathrm{CA}(\psi(\pi))),
$$

for all $\pi \in \mathfrak{S}_{n}(M, I)$. Moreover, $\psi$ fixes $\left|A^{-}(\pi)\right|$.

Proof. Let $M=\left\{m_{1}<\cdots<m_{k}\right\}$ and let $B_{i}$ be the set of entries of $\pi$ that are smaller than and to the left of $m_{i}$. For $S \subseteq[n]$, let $\psi_{S}(\pi)$ be the permutation obtained by reversing the subword of $\pi$ that is a permutation on $S$. Define $\psi$ by

$$
\psi=\psi_{B_{1}} \circ \psi_{B_{2} \cap B_{1}} \circ \cdots \circ \psi_{B_{k-1}} \circ \psi_{B_{k} \cap B_{k-1}} \circ \psi_{B_{k}} .
$$

For instance, with $\pi=125634$ we have $B_{1}=\{1,2,3\}, B_{2}=\{1,2,5\}$ and

$$
\begin{aligned}
\psi(\pi) & =\psi_{B_{1}} \circ \psi_{B_{1} \cap B_{2}} \circ \psi_{B_{2}}(125634) \\
& =\psi_{B_{1}} \circ \psi_{B_{1} \cap B_{2}}(521634) \\
& =\psi_{B_{1}}(512634) \\
& =532614 .
\end{aligned}
$$

It is easy to see that $\psi: \mathfrak{S}_{n}(M, I) \rightarrow \mathfrak{S}_{n}(M, I)$ and that $\psi$ fixes $\left|A^{-}(\pi)\right|$. 
For fixed $y$ we want to show that $\left(\left|A_{y}^{+}\right|,\left|I_{y}^{+}\right|\right) \mapsto\left(\left|I_{y}^{+}\right|,\left|A_{y}^{+}\right|\right)$under $\psi$. If $S$ is a subset of a $B_{j}$ that does not contain $y$ then $\left|A_{y}^{+}\right|$and $\left|I_{y}^{+}\right|$are unchanged under $\psi_{S}$. Let $r$ be the largest index for which $y \in B_{r}$, and let $s$ be the smallest index for which $y \in B_{s}$. Write $\psi$ as $\psi=\alpha \circ \beta \circ \gamma$ where

$$
\beta=\psi_{B_{s}} \circ \psi_{B_{s+1} \cap B_{s}} \circ \cdots \circ \psi_{B_{r-1}} \circ \psi_{B_{r} \cap B_{r-1}} \circ \psi_{B_{r}} .
$$

Now $y$ is not moved, and $\left(\left|A_{y}^{+}\right|,\left|I_{y}^{+}\right|\right)$is conserved, by $\gamma$. Moreover $y$ will remain outside $B_{1} \cup \cdots \cup B_{s-1}$ when we apply $\alpha$ after the action of $\beta \circ \gamma$. Hence it remains to consider the effect on $\left(\left|A_{y}^{+}\right|,\left|I_{y}^{+}\right|\right)$under $\beta$. Suppose $x \in A_{y}^{+}$. Then $\beta$ switches $(x, y)$ an odd number of times. Hence $x \in I_{y}^{+}$after having applied $\beta$. Since $A_{y}^{+} \cup I_{y}^{+} \subseteq B_{s}$, the set $A_{y}^{+} \cup I_{y}^{+}$ remains unchanged under the mappings defining $\beta$. It follows that $\left(A_{y}^{+}, I_{y}^{+}\right) \mapsto\left(I_{y}^{+}, A_{y}^{+}\right)$ under $\beta$. We have proved that $(\mathrm{CA}(\pi), \mathrm{CI}(\pi))=(\mathrm{CI}(\psi(\pi)), \mathrm{CA}(\psi(\pi)))$. By Lemma $9 \psi$ is an involution.

Using a computer we have searched for Mahonian statistics that are of the form $(12, R)+(\pi, S)$, with $\pi \in \mathfrak{S}_{3}, R \subseteq[0,2] \times[0,2]$ and $S \subseteq[0,3] \times[0,3]$. Up to trivial symmetries one new Mahonian statistic apart from MIX was found. Namely

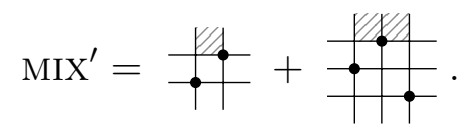

Again, MIX' is, in a sense, a mix of INV and 12. To be more precise, let

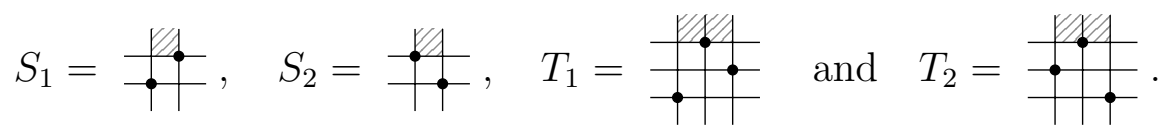

Then $12=S_{1}+T_{1}$, INV $=S_{2}+T_{2}$ and $\mathrm{MIX}^{\prime}=S_{1}+T_{2}$. We note here that $S_{1}$ and $T_{1}$ have appeared before in the literature: $S_{1}$ measure the major cost of the in-situ permutation algorithm $[8,9]$; the statistic INV $+T_{1}$ is identical to lbsum in [5].

Let $\left[\begin{array}{l}n \\ k\end{array}\right]=[n] ! /([k] ![n-k] !)$ denote the usual $q_{1}, q_{2}$-binomial coefficient, where $[n] !=$ $[1][2] \ldots[n]$ and $[n]=q_{1}^{n-1}+q_{1}^{n-2} q_{2}+\cdots+q_{1} q_{2}^{n-2}+q_{2}^{n-1}$. The $q_{1}, q_{2}$-derivative of a function $f(x)$ is defined by

$$
\left(\frac{d}{d x}\right)_{q_{1}, q_{2}} f(x)=\frac{f\left(q_{1} x\right)-f\left(q_{2} x\right)}{q_{1} x-q_{2} x} .
$$

Let

$$
F_{n}=\sum_{\pi \in \mathfrak{S}_{n}} p_{1}^{S_{1}(\pi)} p_{2}^{S_{2}(\pi)} q_{1}^{T_{1}(\pi)} q_{2}^{T_{2}(\pi)}
$$

record the joint distribution of the four permutation statistics $S_{1}, S_{2}, T_{1}$, and $T_{2}$, and let $\mathcal{F}(x)=\sum_{n \geq 0} F_{n} x^{n} /[n]$ ! be the $q_{1}, q_{2}$-exponential generating function for $\left\{F_{n}\right\}_{n \geq 0}$.

Theorem 11. For $n \geq 0$,

$$
F_{n+1}=\sum_{k=0}^{n}\left[\begin{array}{l}
n \\
k
\end{array}\right] p_{1}^{k} p_{2}^{n-k} F_{k} F_{n-k}
$$


Moreover $\left(\frac{d}{d x}\right)_{q_{1}, q_{2}} \mathcal{F}(x)=\mathcal{F}\left(p_{1} x\right) \mathcal{F}\left(p_{2} x\right)$.

Proof. Let $\pi \in \mathfrak{S}_{n+1}$ and write $\pi=L(n+1) R$. Let $A=\operatorname{alph}(L) \subseteq[n]$ be the letters in L. Then

$$
\begin{aligned}
& S_{1}(\pi)=S_{1}(L)+S_{1}(R)+k, \\
& S_{2}(\pi)=S_{2}(L)+S_{2}(R)+n-k, \\
& T_{1}(\pi)=T_{1}(L)+T_{1}(R)+k(n-k)-\gamma(A), \\
& T_{2}(\pi)=T_{2}(L)+T_{2}(R)+\gamma(A),
\end{aligned}
$$

where $k=|A|$ and $\gamma(A)=\{(i, j): i \in A, j \in[n] \backslash A, i>j\}$. Thus

$$
F_{n+1}=\sum_{k=0}^{n}\left(\sum_{A \in\left(\begin{array}{c}
{[n]} \\
k
\end{array}\right)} q_{1}^{k(n-k)-\gamma(A)} q_{2}^{\gamma(A)}\right) p_{1}^{k} p_{2}^{n-k} F_{k} F_{n-k} .
$$

We also have, e.g. from [11, Prop 1.3.17, $\left.m=2, a_{1}=k, a_{2}=n-k\right]$, that

$$
\sum_{A \in\left(\begin{array}{c}
{[n]} \\
k
\end{array}\right)} q_{1}^{k(n-k)-\gamma(A)} q_{2}^{\gamma(A)}=\left[\begin{array}{l}
n \\
k
\end{array}\right],
$$

which proves the recursion formula. The equation for the $q_{1}, q_{2}$-exponential generating function follows.

The fact that MIX' is Mahonian now follows from the symmetry of the variables in the recursion formula.

Corollary 12. The statistic MIX' is Mahonian.

\section{References}

[1] D. André, Développement de sec x and tg x, C. R. Math. Acad. Sci. Paris 88 (1879), 965-979.

[2] E. Babson and E. Steingrímsson, Generalized permutation patterns and a classification of the Mahonian statistics, Sém. Lothar. Combin. 44 (2000) Art. B44b, 18 pp.

[3] F. Bergeron, M. Bousquet-Mélou and S. Dulucq, Standard paths in the composition poset, Ann. Sci. Math. Québec 19(2) (1995), 139-151.

[4] M. Bousquet-Mélou, A. Claesson, M. Dukes and S. Kitaev, (2+2)-free posets, ascent sequences and pattern avoiding permutations, J. Comb. Theory A 117 (2010) 884909. 
[5] M. Dukes and A. Reifegerste, The area above the Dyck path of a permutation, Adv. Appl. Math. 45 (2010), 15-23.

[6] D. Foata and M.-P. Schützenberger, Nombres d'Euler et permutations alternantes, A survey of combinatorial theory (Proc. Internat. Sympos., Colorado State Univ., Fort Collins, Colo., 1971), North-Holland, Amsterdam, pp 173-187, 1973.

[7] D. Foata and V. Strehl, Rearrangements of the symmetric group and enumerative properties of the tangent and secant numbers, Math. Z., 137, (1974), 257-264.

[8] P. Kirschenhofer, H. Prodinger and R. F. Tichy, A contribution to the analysis of in situ permutation, Glas. Mat. Ser. III 22(2) (1987) 269-278.

[9] D. E. Knuth, Mathematical analysis of algorithms, Information Processing 71, North Holland Publishing Company, 1972, Proceedings of IFIP Congress, Ljubljana, 1971, pp. 19-27. Reprinted in: Selected papers on analysis of algorithms, CSLI Publications, Stanford, CA, 2000.

[10] R. Simion and F. W. Schmidt, Restricted Permutations, Europ. J. Combin. 6 (1985), 383-406.

[11] R. P. Stanley, Enumerative combinatorics. Vol. 1., Cambridge University Press, Cambridge, 1997.

[12] R. P. Stanley, A Survey of Alternating Permutations, arXiv:0912.4240.

[13] S. Sundaram, The homology representations of the symmetric group on CohenMacaulay subposets of the partition lattice, Adv. Math. 104 (1994), 225-296.

[14] J. West, Permutations with forbidden subsequences and Stack sortable permutations, Ph.D. thesis, Massachusetts Institute of Technology, 1990. 